\title{
ON ZERO SUBRINGS AND PERIODIC SUBRINGS
}

\author{
HOWARD E. BELL
}

(Received 23 October 2000)

\begin{abstract}
We give new proofs of two theorems on rings in which every zero subring is finite; and we apply these theorems to obtain a necessary and sufficient condition for an infinite ring with periodic additive group to have an infinite periodic subring.
\end{abstract}

2000 Mathematics Subject Classification. 16N40, 16N60, 16P99.

Let $R$ be a ring and $N$ its set of nilpotent elements; and call $R$ reduced if $N=\{0\}$. Following [4], call $R$ an FZS-ring if every zero subring-that is, every subring with trivial multiplication-is finite. It was proved in [1] that every nil FZS-ring is finite-a result which in more transparent form is as follows.

THEOREM 1. Every infinite nil ring contains an infinite zero subring.

Later, in [4], it was shown that every ring with $N$ infinite contains an infinite zero subring. The proof relies on Theorem 1 together with the following result.

THEOREM 2 (see [4]). If $R$ is any semiprime FZS-ring, then $R=B \oplus C$, where $B$ is reduced and $C$ is a direct sum of finitely many total matrix rings over finite fields.

Theorems 1 and 2 have had several applications in the study of commutativity and finiteness. Since the proofs in [1,4] are rather complicated, it is desirable to have new and simpler proofs; and in our first major section, we present such proofs. In our final section, we apply Theorems 1 and 2 in proving a new theorem on existence of infinite periodic subrings.

1. Preliminaries. Let $\mathbb{Z}$ and $\mathbb{Z}^{+}$denote, respectively the ring of integers and the set of positive integers. For the ring $R$, denote by the symbols $T$ and $P(R)$, respectively the ideal of torsion elements and the prime radical; and for each $n \in \mathbb{Z}^{+}$, define $R_{n}$ to be $\left\{x \in R \mid x^{n}=0\right\}$. For $Y$ an element or subset of $R$, let $\langle Y\rangle$ be the subring generated by $Y$; let $A_{l}(Y), A_{r}(Y)$, and $A(Y)$ be the left, right, and two-sided annihilators of $Y$; and let $C_{R}(Y)$ be the centralizer of $Y$. For $x, y \in R$, let $[x, y]$ be the commutator $x y-y x$.

The subring $S$ of $R$ is said to be of finite index in $R$ if $(S,+)$ is of finite index in $(R,+)$. An element $x \in R$ is called periodic if there exist distinct positive integers $m, n$ such that $x^{m}=x^{n}$; and the ring $R$ is called periodic if each of its elements is periodic.

We will use without explicit mention two well-known facts:

(i) the intersection of finitely many subrings of finite index in $R$ is a subring of finite index in $R$; 
(ii) if $R$ is semiprime and $I$ is an ideal of $R$, then $R / A(I)$ is semiprime. We will also need several lemmas.

Lemma 1.1 is a theorem from [6]; Lemma 1.2 appears in [3], and with a different proof in [2]; Lemma 1.3, also given without proof, is all but obvious. Lemma 1.6, which appears to be new, is the key to our proofs of Theorems 1 and 2 .

LEMMA 1.1. If $R$ is a ring and $S$ is a subring of finite index in $R$, then $S$ contains an ideal of $R$ which is of finite index in $R$.

LEMMA 1.2. Let $R$ be a ring with the property that for each $x \in R$, there exist $m \in \mathbb{Z}^{+}$ and $p(t) \in \mathbb{Z}[t]$ such that $x^{m}=x^{m+1} p(x)$. Then $R$ is periodic.

LEMMA 1.3. If $R$ is any ring with $N \subseteq T$ and $H$ is any finite set of pairwise orthogonal elements of $N$, then $\langle H\rangle$ is finite.

LEMMA 1.4. If $R$ is any ring in which $R_{2}$ is finite, then $R$ is of bounded index-that is, $N=R_{n}$ for some $n \in \mathbb{Z}^{+}$.

Proof. Let $M=\left|R_{2}\right|$ and let $x \in N$ such that $x^{2 k}=0$ for $k \geq M+1$; and note that $x^{k}, x^{k+1}, \ldots, x^{2 k-1}$ are all in $R_{2}$. Since $k>M$, these elements cannot be distinct; hence there exist $h, j \in \mathbb{Z}^{+}$such that $h<j \leq 2 k-1$ and $x^{h}=x^{h+m(j-h)}$ for all $m \in \mathbb{Z}^{+}$. It follows that $x^{h}=0$; hence $y^{2 M}=0$ for all $y \in N$.

LEMMA 1.5. If $R$ is any FZS-ring, then $N \subseteq T$.

Proof. Let $R$ be a ring with $N \backslash T \neq \varnothing$, and let $x \in N \backslash T$. Then there exists a smallest $n \in \mathbb{Z}^{+}$such that $x^{n} \in T$, and there exists $k \in \mathbb{Z}^{+}$for which $k x^{n}=0$. Since $k x^{n-1} \notin T$, $\left\langle k x^{n-1}\right\rangle$ is an infinite zero subring of $R$.

LEMMA 1.6. If $R$ is any FZS-ring and $x$ is any element of $N$, then $A(x)$ is of finite index in $R$. Hence, if $S$ is any finite subset of $N, A(S)$ is of finite index in $R$.

Proof. We use induction on the degree of nilpotence. Suppose first that $y^{2}=0$. Define $\Phi: R y \rightarrow R$ by $r y \mapsto[r y, y]=-y r y$; and note that $\Phi(R y)$ is a zero subring of $R$, hence finite. Thus $\operatorname{ker} \Phi=R y \cap C_{R}(y)$ is of finite index in $R y$. But it is easily seen that ker $\Phi$ is a zero ring, hence is finite; consequently, $R y$ is finite. Now consider $\eta: R \rightarrow R y$ defined by $r \mapsto r y$, and note that ker $\eta=A_{l}(y)$ is of finite index in $R$. Similarly, $A_{r}(y)$ is of finite index and so is $A(y)=A_{l}(y) \cap A_{r}(y)$.

Now assume that $A(x)$ is of finite index for all $x \in N$ with degree of nilpotence less than $k$, and let $y \in N$ be such that $y^{k}=0$. Then $A\left(y^{2}\right)$ is of finite index in $R$. Define $\Phi: A\left(y^{2}\right) y \rightarrow R$ by $s y \mapsto[s y, y], s \in A\left(y^{2}\right)$; and note that both $\Phi\left(A\left(y^{2}\right) y\right)$ and $\operatorname{ker} \Phi=A\left(y^{2}\right) y \cap C_{R}(y)$ are zero rings, so that $A\left(y^{2}\right) y$ is finite. Consider the map $\Psi=A\left(y^{2}\right) \rightarrow A\left(y^{2}\right) y$ given by $s \rightarrow s y$. Now $\operatorname{ker} \Psi=A\left(y^{2}\right) \cap A_{l}(y)$ must be of finite index in $A\left(y^{2}\right)$; and since $A\left(y^{2}\right)$ is of finite index in $R$, $\operatorname{ker} \Psi$ is of finite index in $R$. It follows that $A_{l}(y)$ is of finite index in $R$; and a similar argument shows that $A_{r}(y)$ is of finite index in $R$. Therefore $A(y)$ is of finite index in R.

LEMMA 1.7. Let $p$ be a prime, and let $R$ be a ring such that $p R=\{0\}$.

(i) If $a \in R$ and $a^{p^{k}}=a$, then $a^{p^{m k}}=a$ for all $m \in \mathbb{Z}^{+}$. Hence if $a, b \in R$ with $a^{p^{k}}=a$ and $b^{p^{j}}=b$, there exists $n \in \mathbb{Z}^{+}$such that $a^{p^{n}}=a$ and $b^{p^{n}}=b$. 
(ii) If $a \in R$ and $a^{p^{k}}=a$, then for each $s \in \mathbb{Z},(s a)^{p^{k}}=s a$.

(iii) If $R$ is reduced and $a$ is a periodic element of $R$, then there exists $n \in \mathbb{Z}^{+}$such that $a^{p^{n}}=a$.

Proof. (i) is almost obvious, and (ii) follows from the fact that $s^{p} \equiv s(\bmod p)$ for all $s \in \mathbb{Z}$. To obtain (iii), note that if $R$ is reduced and $a$ is periodic, then $\langle a\rangle$ is finite, hence a direct sum of finite fields, necessarily of characteristic $p$. Since $\operatorname{GF}\left(p^{\alpha}\right)$ satisfies the identity $x^{p^{\alpha}}=x$, the conclusion of (iii) follows by (i).

\section{Proofs of Theorems 1 and 2}

Proof of Theorem 1. Suppose $R$ is a counterexample. Note that $R$ is an $F Z S$-ring, so $R=T$ by Lemma 1.5. It is easy to see that $R$ contains a maximal finite zero subring $S$. By Lemma 1.6, $A(S)$ is infinite; and maximality of $S$ forces $A(S)_{2}=S$. Thus, by replacing $R$ by $A(S)$, we may assume that $R_{2}$ is finite.

By Lemma 1.6, we can construct infinite sequences of pairwise orthogonal elements; and by Lemma 1.4 there is a smallest $M \in \mathbb{Z}^{+}$for which $R_{M}$ contains such sequences. Let $u_{1}, u_{2}, \ldots$ be an infinite sequence of pairwise orthogonal elements of $R_{M}$. Using Lemma 1.3, we can refine this sequence to obtain an infinite subsequence $v_{1}, v_{2}, \ldots$ such that for each $j \geq 2, v_{j} \notin\left\langle v_{1}, v_{2}, \ldots, v_{j-1}\right\rangle$. Defining $V_{0}$ to be $\left\{v_{j}^{2} \mid j \in \mathbb{Z}^{+}\right\}$, we see that $V_{0} \subseteq R_{M-1}$ and hence $V_{0}$ is finite, so we may assume without loss of generality that there exists a single $s \in R$ such that $v_{j}^{2}=s$ for all $j \in \mathbb{Z}^{+}$. Take $m \in \mathbb{Z}^{+}$such that $m s=0$; and for each $j \in \mathbb{Z}^{+}$, define $w_{j}=\sum_{i=1}^{m j} v_{i}$. Then the $w_{j}$ form an infinite subset of $R_{2}$, contrary to the fact that $R_{2}$ is finite. The proof is now complete.

Proof of TheOrem 2. As before, since $R$ is an FZS-ring, there is a maximal finite zero subring $S$; and by Lemma $1.6 A(S)$ is of finite index in $R$. By Lemma 1.1, $A(S)$ contains an ideal $I$ of $R$ which is also of finite index in $R$. Let $C=A(I)$ and let $B=A(C)$. Then $B \supseteq I$, so $B$ is of finite index in $R$.

Next we show that $B$ is reduced. Let $x \in B$ such that $x^{2}=0$. Then $x \in A(C)$; and since $S \subseteq C$, the maximality of $S$ forces $x \in B \cap C=\{0\}$. Therefore, $B$ is reduced.

The rest of the proof is as in [4]. Since $R / B$ is finite and semiprime, we can write it as $M_{1} \oplus \cdots \oplus M_{k}$, where the $M_{i}$ are total matrix rings over finite fields. Let $C^{\prime}=(B+C) / B$ and note that $C^{\prime}$ is an ideal of $R / B$ and $C^{\prime} \cong C$. Now $C^{\prime}$ must be a direct sum of some of the $M_{i}$, so $R / B=C^{\prime} \oplus D^{\prime}$ where $D^{\prime}$ is the annihilator of $C^{\prime}$. Taking $D$ to be an ideal of $R$ containing $B$ for which $D / B=D^{\prime}$, and noting that $C^{\prime} D^{\prime}=\{0\}$, we have $C D \subseteq B$. But $C D \subseteq C$ as well, so $C D \subseteq B \cap C=\{0\}$ and $D \subseteq A(C)=B$; therefore $D^{\prime}=\{0\}$ and $C^{\prime}=R / B$. It follows that $R=B+C$ and hence $R=B \oplus C$; and since $C \cong C^{\prime}, C$ is a direct sum of total matrix rings as required.

REMARK 2.1. In [5], Lanski established the conclusion of Theorem 2 under the apparently stronger hypothesis that $N$ is finite; and his proof uses induction on $|N|$. As we noted in the introduction, it follows from Theorems 1 and 2 that $R$ is an FZS-ring if and only if $N$ is finite.

3. A theorem on periodic subrings. We have noted that if $N$ is infinite, $R$ contains an infinite nil subring. Since periodic elements extend the notion of nilpotent element, 
it is natural to ask whether there is a periodic analogue-that is, to ask whether a ring with infinitely many periodic elements must have an infinite periodic subring. The answer in general is no, even in the case of commutative rings. The complex field $\mathbb{C}$ is a counterexample, for the set of nonzero periodic elements is the set $U$ of roots of unity, and $u \in U$ implies $2 u \notin U$. Moreover, if $S$ is any finite ring, $\mathbb{C} \oplus S$ is also a counterexample; therefore, we restrict our attention to rings $R$ for which $R=T$.

THEOREM 3.1. Let $R$ be a ring with $R=T$. Then a necessary and sufficient condition for $R$ to have an infinite periodic subring is that $R$ contains an infinite set of pairwisecommuting periodic elements.

Proof. It is known that in any infinite periodic ring $R$, either $N$ is infinite or the center $Z$ is infinite [4, Theorem 7]. Therefore our condition is necessary.

For sufficiency, suppose that $R$ has infinitely many pairwise-commuting periodic elements. Now $R$ is the direct sum of its $p$-primary components $R^{(p)}$; and if there exist infinitely many primes $p_{1}, p_{2}, p_{3}, \ldots$ such that $R^{\left(p_{i}\right)}$ contains a nonzero periodic element $a_{p_{i}}$, then the direct sum of the rings $\left\langle a_{p_{i}}\right\rangle$ is an infinite periodic subring. Thus, we may assume that only finitely many $R^{(p)}$ contain nonzero periodic elements, so we need only consider the case that $R=R^{(p)}$ for some prime $p$. Of course we may assume that $R$ is an FZS-ring.

Consider the factor ring $\bar{R}=R / P(R)$. Since $R$ is an FZS-ring, it follows from Theorem 1 that $P(R)$ is finite, in which case $\bar{R}$ inherits our hypothesis on pairwisecommuting periodic elements. If $\bar{R}$ has an infinite periodic subring $\bar{S}$ and $S$ is its preimage in $R$, then for all $x \in S$, there exist distinct $m, n \in \mathbb{Z}^{+}$such that $x^{n}-x^{m} \in$ $P(R) \subseteq N$; hence $S$ is periodic by Lemma 1.2. Thus, we may assume that $R=R^{(p)}$ and that $R$ is a semiprime $F Z S$-ring.

By Theorem 2, write $R=B \oplus C$, where $B$ is reduced and $C$ is finite; and note that $B$ must have an infinite subset $H$ of pairwise-commuting periodic elements. Note also that $p B=\{0\}$, since $B$ is reduced. Let $a, b \in H$, and by Lemma 1.7(i) and (iii) obtain $n \in \mathbb{Z}^{+}$such that $a^{p^{n}}=a$ and $b^{p^{n}}=b$. It follows at once that $(a-b)^{p^{n}}=a^{p^{n}}-b^{p^{n}}=$ $a-b$ and $(a b)^{p^{n}}=a^{p^{n}} b^{p^{n}}=a b$; and these facts, together with Lemma 1.7(ii) imply that $\langle H\rangle$ is an infinite periodic subring of $R$.

ACKNOWLEDGEment. This research was supported by the Natural Sciences and Engineering Research Council of Canada, Grant No. 3961.

\section{REFERENCES}

[1] H. E. Bell, Infinite subrings of infinite rings and near-rings, Pacific J. Math. 59 (1975), no. 2, 345-358. MR 52\#8197. Zbl 0315.17006.

[2] - On commutativity of periodic rings and near-rings, Acta Math. Acad. Sci. Hungar. 36 (1980), no. 3-4, 293-302. MR 82h:16025. Zbl 0464.16026.

[3] M. Chacron, On a theorem of Herstein, Canad. J. Math. 21 (1969), 1348-1353. MR 41\#6905. Zbl 0213.04302.

[4] A. A. Klein and H. E. Bell, Rings with finitely many nilpotent elements, Comm. Algebra 22 (1994), no. 1, 349-354. MR 94k:16033. Zbl 0806.16017.

[5] C. Lanski, Rings with few nilpotents, Houston J. Math. 18 (1992), no. 4, 577-590. MR 94c:16026. Zbl 0821.16020. 
[6] J. Lewin, Subrings of finite index in finitely generated rings, J. Algebra 5 (1967), 84-88. MR 34\#196. Zbl 0143.05303.

Howard E. Bell: Department of Mathematics, Brock University, St. Catharines, ONTARIO, CANADA L2S 3A1

E-mail address: hbe11@spartan.ac.brocku.ca 


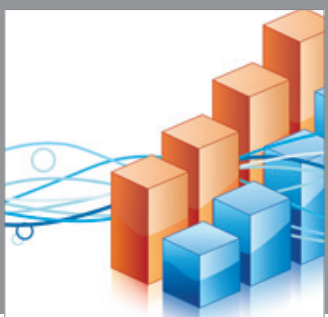

Advances in

Operations Research

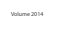

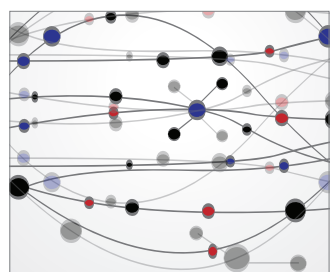

\section{The Scientific} World Journal
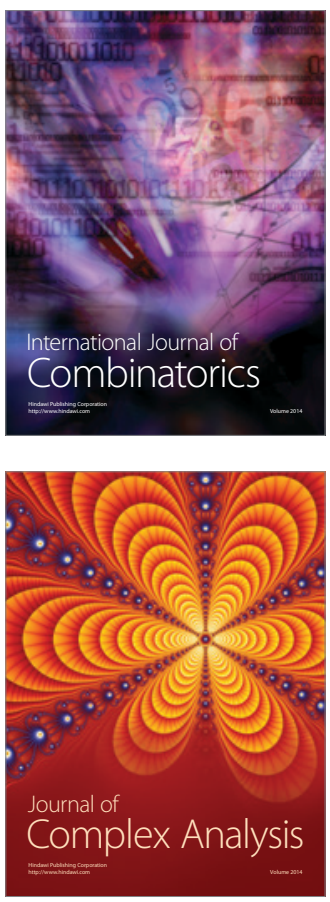

International Journal of

Mathematics and

Mathematical

Sciences
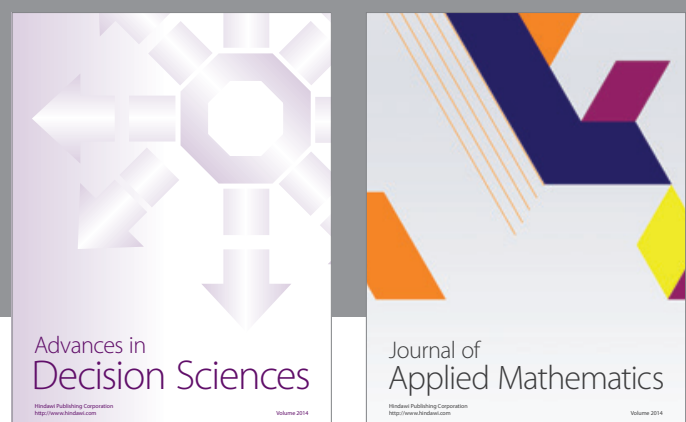

Journal of

Applied Mathematics
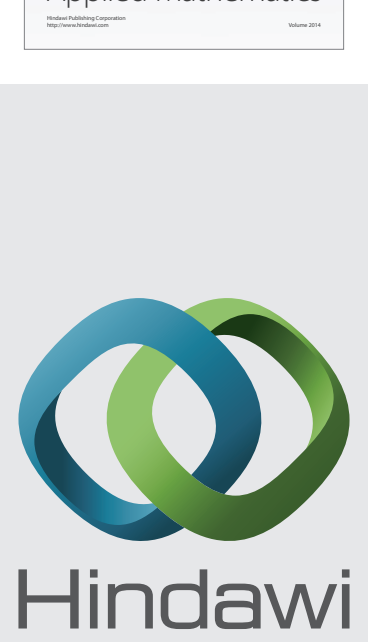

Submit your manuscripts at http://www.hindawi.com
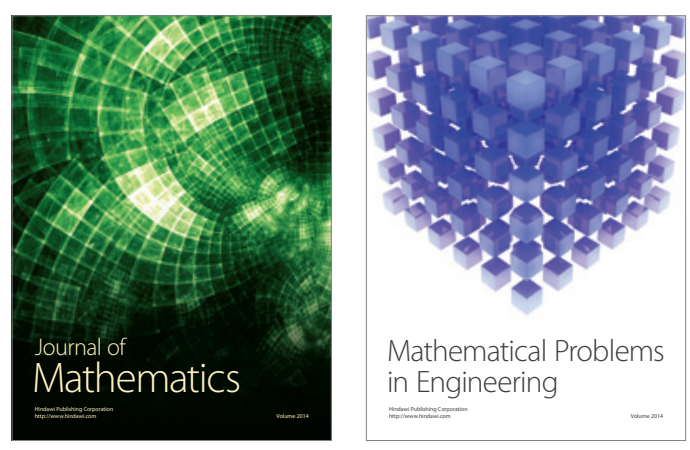

Mathematical Problems in Engineering
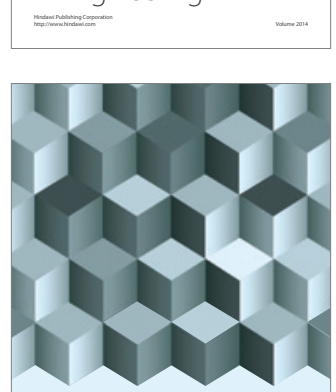

Journal of

Function Spaces
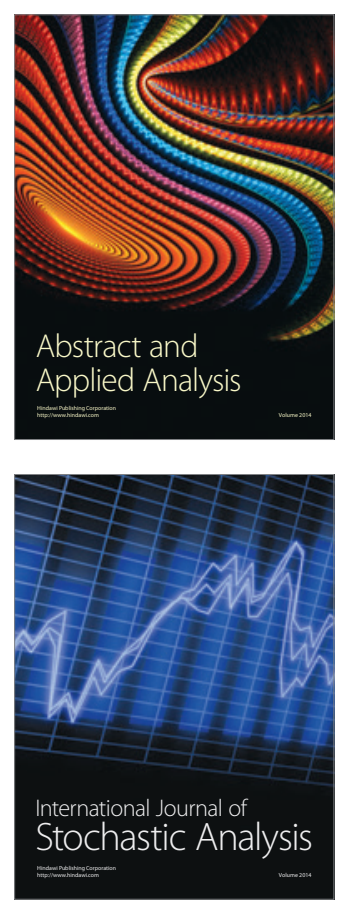

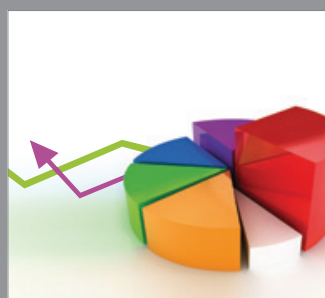

ournal of

Probability and Statistics

Promensencen
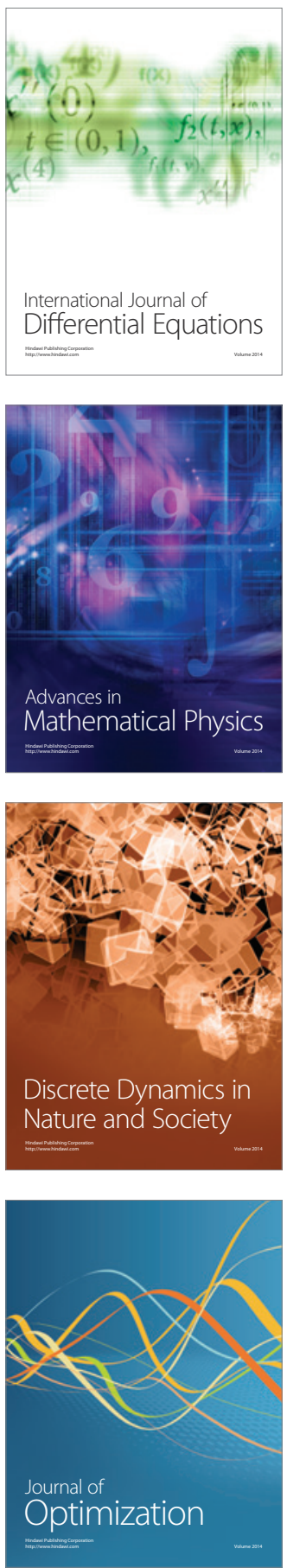